\title{
Enrico Gavalotti, Il professorino. Giuseppe Dossetti tra crisi del fascismo e costruzione della democrazia 1940-1948
}

Bologna, Il Mulino, 2013, 885 p.

Jean-Dominique Durand

\section{OpenEdition \\ Journals}

\section{Édition électronique}

URL : http://journals.openedition.org/assr/26415

DOI : $10.4000 /$ assr. 26415

ISSN : $1777-5825$

\section{Éditeur}

Éditions de l'EHESS

Édition imprimée

Date de publication : 31 décembre 2014

Pagination : 188

ISBN : 978-2-7132-2467-6

ISSN : 0335-5985

\section{Référence électronique}

Jean-Dominique Durand, «Enrico Gavalotti, II professorino. Giuseppe Dossetti tra crisi del fascismo e costruzione della democrazia 1940-1948», Archives de sciences sociales des religions [En ligne], 168 | 2014, mis en ligne le 17 avril 2015, consulté le 22 septembre 2020. URL : http://

journals.openedition.org/assr/26415; DOI : https://doi.org/10.4000/assr.26415 


\section{Enrico Gavalotti, Il professorino. Giuseppe Dossetti tra crisi del fascismo e costruzione della democrazia 1940-1948}

Bologna, Il Mulino, 2013, 885 p.

Jean-Dominique Durand

RÉFÉRENCE

Enrico Gavalotti, Il professorino. Giuseppe Dossetti tra crisi del fascismo e costruzione della democrazia 1940-1948, Bologna, Il Mulino, 2013, 885 p. 
1 Giuseppe Dossetti (1913-1996) fut l'une des plus grandes figures du catholicisme italien au $\mathrm{xx}^{\mathrm{e}}$ siècle. Il a rassemblé en lui plusieurs types d'engagements, qui se sont toujours imbriqués les uns dans les autres: l'engagement politique, l'engagement culturel, l'engagement ecclésial. L'engagement politique, ce fut d'abord celui du jeune professeur de Droit canon au temps du fascisme et de la découverte de Jacques Maritain qui ne devait jamais cesser de l'éclairer ; celle-ci nourrit alors un rêve de démocratie régénérée par le christianisme. En 1943, il entra en résistance à la suite de l'invasion allemande, il connut la clandestinité et la lutte armée dans une région, l'ÉmilieRomagne, marquée par une forte présence

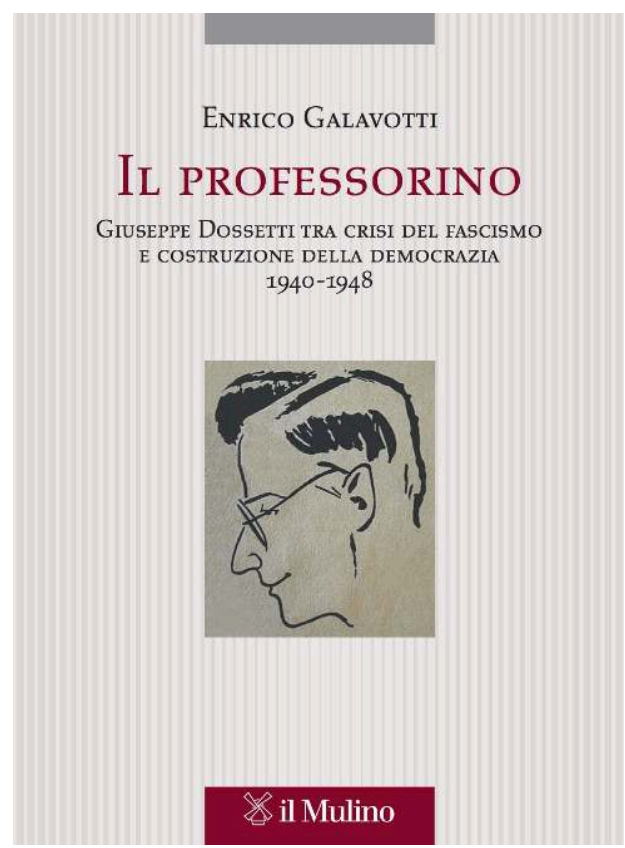
communiste, expérience qui le marqua à jamais. À la libération, le résistant participa à la reconstruction du pays au sein du parti démocrate chrétien, dont il fut vice-secrétaire. Élu à l'Assemblée constituante en 1946, il fut l'un des principaux rédacteurs de la Constitution républicaine. Mais il ne se reconnut pas dans l'action politique au quotidien; entré en conflit avec Alcide De Gasperi, il préconisait l'entente avec les forces populaires, sans jamais perdre sa lucidité sur les réalités du Parti communiste. Il se retira de l'action politique en 1956, pour devenir prêtre en 1959 et fonder une congrégation, la Piccola Famiglia dell'Annunziata. Très proche du cardinal Lercaro, archevêque de Bologne, il joua également un rôle majeur au Concile Vatican II. Son engagement sur le plan de la culture fut lui aussi foisonnant. Il fonda à Bologne en 1953, le Centre de documentation pour les études religieuses, devenu ensuite l'Istituto per le Scienze Religiose, aujourd'hui Fondation qui porte le nom de Jean XXIII. Il s'agissait de doter le catholicisme italien d'un instrument de recherche fondé sur une puissante bibliothèque, capable de promouvoir la rénovation de l'Église par la culture.

C'est dans le cadre de cet institut qu'Enrico Gavalotti, par ailleurs professeur d'Histoire du christianisme à l'Université de Chieti-Pescara, développe ses recherches. Il construit une biographie de Dossetti en plusieurs volumes, qui constitue une véritable somme. Il a publié en 2006 un premier ouvrage fort documenté sur la jeunesse et les années de formation (Il giovane Dossetti. Gli anni della formazione 1913-1939, Bologne, Il Mulino, 250 p.). Aujourd'hui, il étudie les années cruciales de cette vie si riche, les années qui vont de 1940 à 1948, entre crise du fascisme et construction de la démocratie. Ce sont les années de la crise de l'Italie marquées par la guerre de Mussolini qui se révéla vite désastreuse, l'effondrement du régime, le débarquement allié en Sicile et l'occupation de l'Italie par les Allemands, l'interminable libération du territoire qui ne s'achève qu'en mai 1945, le désastre de trois formes de conflit, la guerre sur le territoire italien avec les bombardements et les destructions, une occupation nazie particulièrement cruelle, une guerre civile avec ses exactions et ses cruautés. Ce sont les années de reconstruction d'un pays ravagé sur le plan matériel et économique, mais aussi moral 
et spirituel, où deux forces opposées émergent et jouent un rôle déterminant, le parti communiste et la Démocratie chrétienne soutenue par les puissantes structures de l'Église catholique (diocèses, paroisses, mouvements, congrégations religieuses) sous l'autorité du pape.

Le fort volume que donne Enrico Gavalotti retrace quasiment au jour le jour l'évolution de Dossetti dans ces huit années pleines de fureur. L'entrée en guerre de l'Italie le 10 juin 1940 marqua une rupture, « la rupture avec la sérénité précédente » (p. 27), et le début d'une nouvelle réflexion politique. En même temps il poursuivait ses études en Droit canonique et ecclésiastique, pour enseigner, à partir de 1942 à l'Université de Modène, et à l'Université du Sacré-Cœur de Milan. Il participait avec de jeunes collègues comme Giorgio La Pira à des réunions pour réfléchir à l'avenir de l'Italie, et développa la conviction de l'incompatibilité entre christianisme et totalitarisme. Il participa à la préparation d'un long commentaire du Radiomessage de Pie XII de Noël 1942, qui eut un grand retentissement dans le catholicisme italien. Engagé dans la résistance dans la région de Reggio Emilia, il fit partie du Comité de Libération Nationale provincial. Bien qu'hostile à la création d'un parti catholique («parti et catholique sont deux termes en contradiction» disait-il, p.150), il se rallia à la Démocratie chrétienne. Mais son rêve, partagé par de nombreux catholiques, était plutôt une vaste formation du type du Labour Party, rassemblant chrétiens et nonchrétiens. Vice-secrétaire de la DC, il se sentit toujours mal à l'aise dans une confrontation politique de plus en plus dure avec la gauche. Au moment du référendum institutionnel, il milita en faveur de la République lorsque son parti, pour ménager les sensibilités plutôt monarchistes des catholiques et d'une grande partie de l'épiscopat, choisit la neutralité. C'est surtout à l'Assemblée constituante que Dossetti donna toute sa dimension. Comme juriste, il se sentit tout de suite très à l'aise dans les débats de l'assemblée. Il constitua avec ses amis de l'Université catholique de Milan, Giorgio La Pira, Amintore Fanfani, Giuseppe Lazzati notamment, les "professorini », terme qui n'avait rien de péjoratif, qui reconnaissait au contraire l'influence de ces jeunes intellectuels, un groupe actif, au sein des réseaux catholiques avec l'association Civitas Humana puis la revue Cronache Sociali, et au sein de l'Assemblée pour en orienter les débats. À Rome, ils habitaient ensemble, abrités à la Chiesa Nuova, au point que l'on a pu parler de la communauté du Porcellino et d'un «conventino» (p.692). Enrico Gavalotti montre comment Dossetti exerça un leadership déterminant pour orienter la constitution italienne, pour placer la personne humaine au centre de la construction du nouvel État, n'hésitant pas à travailler avec les communistes, par exemple sur l'article devant traiter de l'utilité sociale du travail (p. 495-498), ou pour convaincre ceux-ci de la nécessité d'accepter le principe concordataire sur le sujet des relations entre l'Église et l'État. L'auteur montre aussi fort bien l'insertion de Dossetti dans l'Église d'Italie, ses relations avec la Secrétairerie d'État, son influence dans la rédaction de la constitution apostolique Provida Mater sur les Instituts séculiers. Mais toujours mal à l'aise dans le jeu politique, il fallut l'intervention de Pie XII lui-même pour qu'il acceptât d'être candidat aux élections législatives d'avril 1948 (p. 819). L'Italie entrait alors dans une nouvelle phase de son histoire, marquée par l'hégémonie catholique jusqu'en 1992.

4 L'ouvrage de Gavalotti, avec ses 850 pages de texte à la typographie serrée, est foisonnant. C'est un grand livre savant qui donne à connaître non seulement le personnage de Dossetti, mais à travers lui, l'histoire du catholicisme italien, donc de l'Italie, dans ces années de crise qui ont permis une vraie reconstruction démocratique 
à l'issue de vingt années de fascisme et d'une guerre effroyable. Fondé sur toutes les archives disponibles, c'est un ouvrage majeur dans l'historiographie de l'Italie. 